\title{
Achromatic lens systems for near infrared instruments
}

\section{Performances and limitations of standard Flint glasses}

\author{
E. Oliva and S. Gennari \\ Osservatorio Astrofisico di Arcetri, Largo E. Fermi 5, I-50125 Firenze, Italy
}

Received May 5; accepted July 22, 1997

\begin{abstract}
This paper investigates which normal Flint glasses are best suited for the design of lens systems working in the infrared up to about $1.7 \mu \mathrm{m}$, and possibly up to $2.5 \mu \mathrm{m}$. Between 0.9 and $2.5 \mu \mathrm{m}$ the best known achromatic pairs are $\mathrm{BaF}_{2}-\mathrm{IRG} 2$ and $\mathrm{SrF}_{2}-\mathrm{IRG} 3$ and, to a lesser extent, $\mathrm{CaF}_{2}-\mathrm{IRG} 7$ (Oliva \& Gennari 1995). Unfortunately, Schott will most probably stop the production of these very little used and commercially uninteresting IRG glasses.

Here we show that equally good performances can be obtained coupling $\mathrm{BaF}_{2}$ or $\mathrm{SrF}_{2}$ with standard $\mathrm{SF}$ glasses. The pairs we analyze in details are $\mathrm{BaF}_{2}-\mathrm{SF} 6$ and $\mathrm{BaF}_{2}-\mathrm{SF} 56 \mathrm{~A}$, and we also present new measurements of transmission for several SF glasses which are quite transparent up to $1.65 \mu \mathrm{m}$ and can be therefore readily employed in fiber-fed spectrographs and other instruments which do not extend beyond the $\mathrm{H}$ atmospheric window. At longer wavelengths the use of SF glasses is limited by strong water absorption features, but these could be eliminated by preparing the glass in vacuum environment. Although this production is expensive and commercially unattractive, we hope however that a large enough group of astronomers will support glass manufacturers in the production of "IR-grade SF glasses".

As a practical application we present representative results of the design of F/2 (4 lenses) and F/1.4 ( 5 lenses) cameras for near infrared $(0.95-2.5 \mu \mathrm{m})$ spectrometers.
\end{abstract}

Key words: instrumentation: miscellaneous instrumentation: spectrographs

\section{Introduction}

The optical design of dioptric systems working in the infrared is complicated by several factors. The lenses must be cooled to cryogenic temperatures, this prevents using

Send offprint requests to: E. Oliva cemented doublets/triplets and the only way to obtain a system with good total transmission is using as few lenses as possible. The choice of optical materials is limited by the fact that "normal" glasses are quite opaque in the infrared due to absorption bands of water which is normally bound in the glass matrix, and sometimes because of absorption intrinsic to the glassy compounds. For this reason normal glasses were mostly ignored, and the choice of optical materials was traditionally limited to a few crystals such as $\mathrm{CaF}_{2}, \mathrm{ZnS}, \mathrm{ZnSe}$ and $\mathrm{BaF}_{2}$. Prior to the "re-discovery" of Schott IR glasses (Delabre 1994; Oliva \& Gennari 1995) the only glassy material employed in near IR astronomical instrument was IR-grade fused silica. This glass has high dispersion (i.e. is a good Flint) but has a very high partial dispersion (cf. Fig. 1) and is not suited to produce good achromatic pairs with any of the Crowns known.

Normal alkaline-earth fluoride crystals have very low dispersion in the near infrared (cf. Fig. 1). The main problem faced by those designing IR lenses instruments is therefore to find a high dispersive ("Flint") material to couple with $\mathrm{BaF}_{2}, \mathrm{SrF}_{2}$ or $\mathrm{CaF}_{2}$. The condition for achromatism requires that the Flint should have a partial dispersion similar to the alkaline-earth fluoride crystals, and this is not satisfied by any of the crystalline materials commonly used. However, an excellent match is provided by the Schott IRG glasses which were recently employed in several astronomical instruments working up to $2.5 \mu \mathrm{m}$ (e.g. Oliva \& Gennari 1995). Unfortunately, the only people who seem interested in IRG2, IRG3 and IRG7 are astronomers, and there is hardly any commercial profit for Schott to continue the production of these glasses. Finding alternatives to the IRG glasses is the main aim of this paper which is also intended to give relatively simple solutions for the design of very fast cameras for IR spectrographs.

In Sect. 2 we analyze the chromatic characteristics of normal SF glasses, define the best Flints to couple to $\mathrm{BaF}_{2}, \mathrm{SrF}_{2}$, and briefly discuss the uncertainties on the variation of their refraction indices with temperature. 


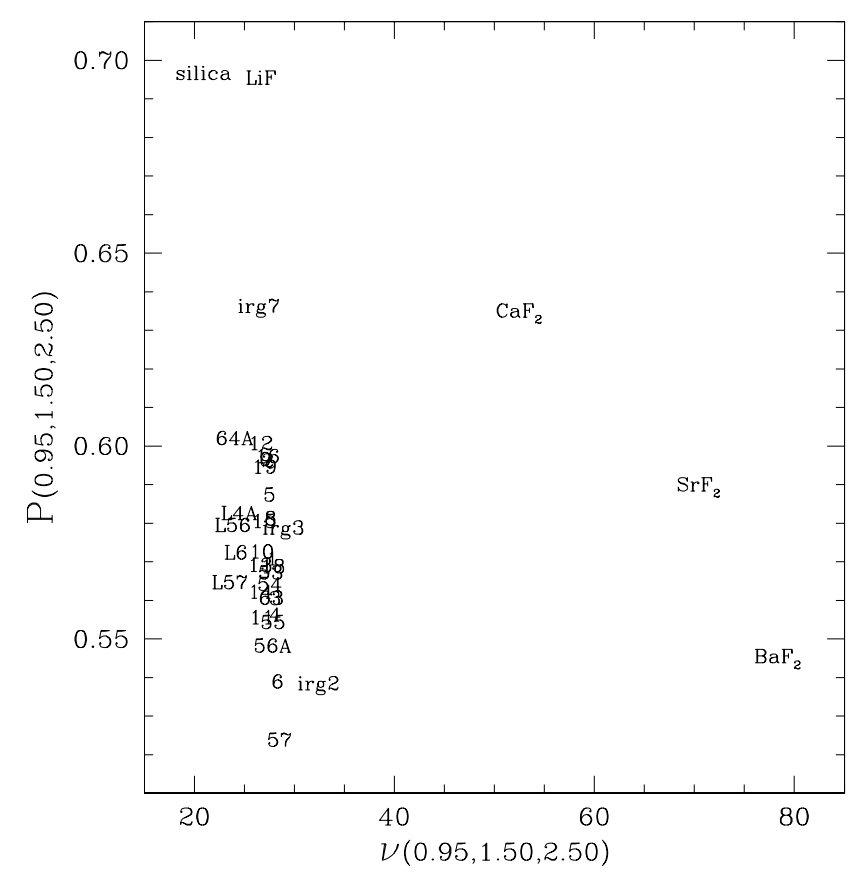

Fig. 1. Plot of the NIR partial dispersion $P=[n(1.5)-n(2.5)] /[n(0.95)-n(2.5)]$ versus the "NIR Abbe number" $\nu=[n(1.5)-1] /[n(0.95)-n(2.5)]$ Achromatic pairs are those with very similar $P$ and large $\Delta \nu$. For clarity, the glass names are without the prefix "SF"

Representative designs of fast cameras for IR spectrographs are discussed in Sect. 3. New measurements of the IR absorption of SF glasses are presented in Sect. 4.

\section{The selection of Flint glasses}

A convenient way to identify the Flint glasses which couple with the alkaline-earth fluoride Crowns is the standard partial dispersion versus Abbe number plot which is displayed in Fig. 1. The most striking result is that SF6 and IRG2 have very similar chromatic characteristics, while SF5 seems an even better match to $\mathrm{SrF}_{2}$ than IRG3. Other interesting pairs are $\mathrm{BaF}_{2}-\mathrm{SF} 56 \mathrm{~A}$ and $\mathrm{SrF}_{2}-\mathrm{SFL} 4 \mathrm{~A}$.

Here we concentrate on the Flint glasses which couple with $\mathrm{BaF}_{2}$. Refraction indices and dispersions at room temperatures are listed in the upper panel of Table 1. The values of $n$ for SF glasses are computed using the Sellmeier's parameters of the Schott catalog which interpolate measurements from 0.37 to $2.33 \mu \mathrm{m}$, the extrapolation to $2.5 \mu \mathrm{m}$ should not introduce significant errors.

\subsection{Temperature dependence of refraction indices}

Table 1 (lower panel) also includes optical parameters at cryogenic temperatures. The values for $\mathrm{BaF}_{2}$ are based on the accurate measurements by Feldman et al. (1979) which tabulate $\mathrm{d} n / \mathrm{d} T$ at $0.46,0.63,1.15,3.39,10.6 \mu \mathrm{m}$ and many temperatures between +200 and $-180{ }^{\circ} \mathrm{C}$. The

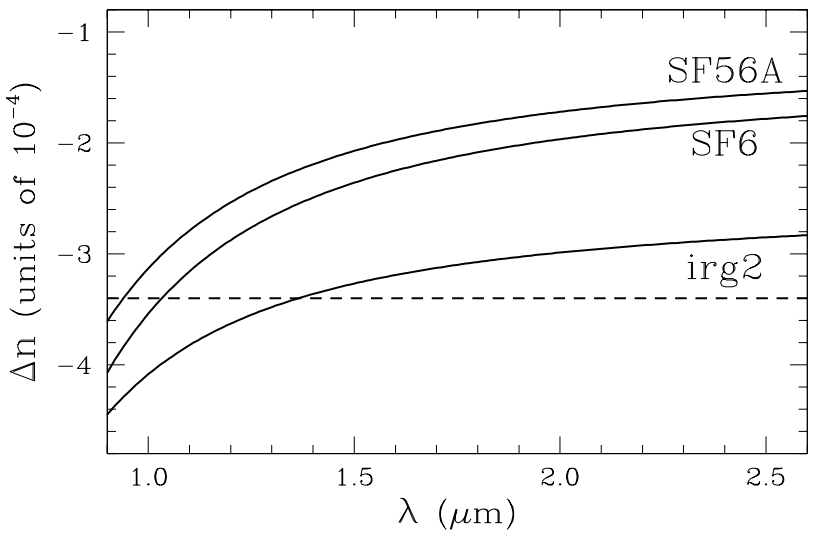

Fig. 2. Solid lines: predicted variation of refractive index between room and cryogenic temperatures $(\Delta n=$ $\left.n\left(-200{ }^{\circ} \mathrm{C}\right)-n\left(25^{\circ} \mathrm{C}\right)\right)$. The curves are based on the $T$-dependent, Sellmeier equation (Eq. 2) whose parameters are taken from the Schott catalog (cf. Sect. 2). The dashed lines is the value for IRG2 estimated by Doyon et al. (1995) and also confirmed within $\pm 10^{-4}$ by direct measurements (van Dijsseldonk 1995)

resulting refraction indices at $-200{ }^{\circ} \mathrm{C}$ can be conveniently fitted with a two terms Sellmeier's formula which yields

$$
\begin{aligned}
n\left(\mathrm{BaF}_{2}-200{ }^{\circ} \mathrm{C}\right)=\frac{1.158534 \lambda^{2}}{\lambda^{2}-.0846639^{2}}+ & \frac{4.056181 \lambda^{2}}{\lambda^{2}-47.54707^{2}} \\
& \lambda \geq 0.46 \mu \mathrm{m} . \quad(1)
\end{aligned}
$$

For the Flint glasses we use the Schott formula

$$
\begin{aligned}
& \Delta n(\lambda, T)=\frac{n^{2}\left(\lambda, T_{0}\right)-1}{2 n\left(\lambda, T_{0}\right)}\left(D_{0} \Delta T+D_{1} \Delta T^{2}+D_{2} \Delta T^{3}\right. \\
& \left.+\frac{E_{0} \Delta T+E_{1} \Delta T^{2}}{\lambda^{2}-\lambda_{\mathrm{TK}}^{2}}\right)
\end{aligned}
$$

and the $D_{0}, D_{1}, D_{2}, E_{0}, E_{1}, \lambda_{\mathrm{TK}}$ coefficients from the Schott catalog which are based on data at $\lambda \leq 1.014 \mu \mathrm{m}$ and $T \geq-100{ }^{\circ} \mathrm{C}$. The values in Table 1 are therefore extrapolated to wavelengths and temperatures for which no measurement exists. However, this should not necessarily introduce large errors because Eq. (2) is based on a physical representation of the glass properties (i.e. the Sellmeier's formula) and the only important uncertainty could be that Eq. (2) does not include the long wavelength resonance which for $\mathrm{SF}$ glasses lies at $\simeq 11 \mu \mathrm{m}$.

The predicted total variation of refraction index with wavelength is plotted in Fig. 2 where we also include IRG2, a glass for which other estimates exist in the literature (see the caption of Fig. 2). The main result is that $\mathrm{d} n / \mathrm{d} T$ is small for glasses, the variation of dispersion is also very low and in practice negligible. This is confirmed by our detailed designs (Sect. 3) where we find that the same systems also give excellent images if the SF glasses have $\Delta n=-210^{-4}$ at all wavelengths, the only modification required is to refocus the array by a few $\times 10 \mu \mathrm{m}$. 


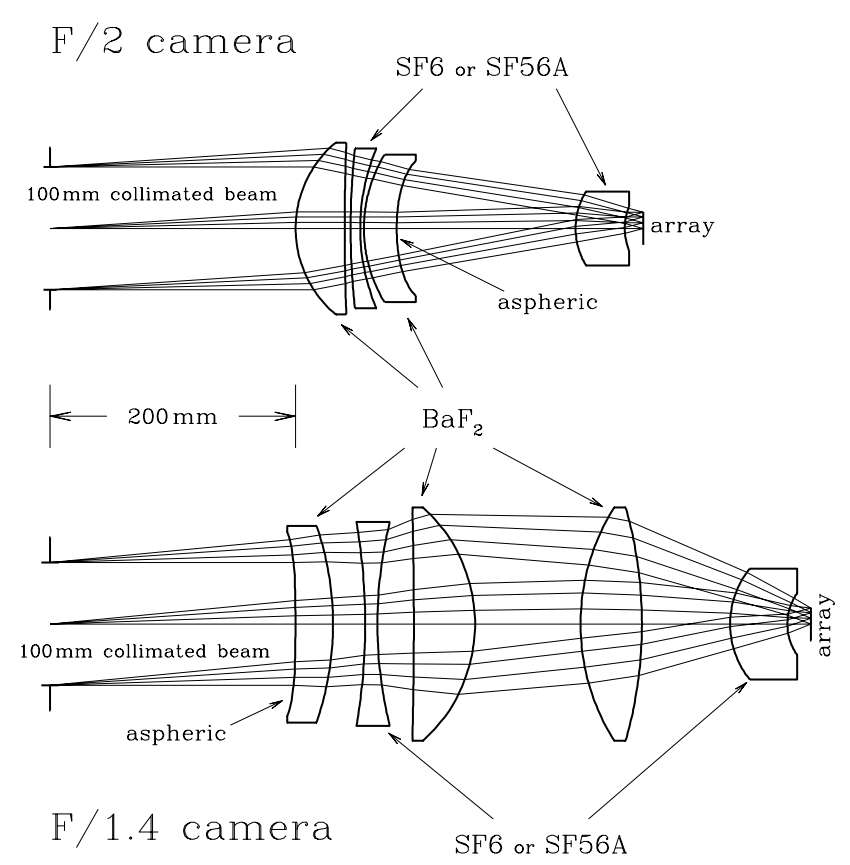

Fig. 3. Layout and ray tracing of $F / 2$ and F/1.4 near-IR cameras. Spot diagrams and encircled energies are displayed in Figs. 4,5 while the details of the design are listed in Table 2

In short, the information presently available on $\mathrm{SF}$ glasses is probably enough for their use in cryogenic lens systems. However, new measurements of refraction indices at $\simeq 70 \mathrm{~K}$ and $\mathrm{IR}$ wavelengths would be useful, and could be essential for the design of multi-objective systems which do not foresee the possibility of refocusing the array.

\section{The design of fast cameras for IR spectrographs}

The combination of large telescopes and IR arrays with small pixels requires that IR spectrometers with a reasonably high throughput (i.e. with few pixels sampling a $1^{\prime \prime}$ slit) must have very fast cameras. For example, an instrument equipped with the Rockwell $1024^{2}$ array $(18.5 \mu \mathrm{m}$ pixel size) and intended to work on a $8 \mathrm{~m}$ class telescope with a $1^{\prime \prime}$ slit sampled by 4 pixels must have a $\mathrm{F} / 2$ camera, while a much more extreme $\mathrm{F} / 1.4$ system is necessary to obtain 3 pixels/arcsec. The results presented here indicate that these types of cameras are relatively easy to design when $\mathrm{BaF}_{2}-\mathrm{SF}$ pairs are adopted.

Representative solutions for $\mathrm{F} / 2$ and $\mathrm{F} / 1.4$ systems are shown in Fig. 3, the spot diagrams and encircled energies are displayed in Figs. 4, 5 while the details of the lenses are listed in Table 2. In these designs we assumed a "typical" $\oslash 100 \mathrm{~mm}$ collimated (parallel) beam allowing for about $200 \mathrm{~mm}$ free space between the pupil image and the first optical surface. Note that the cameras were not optimized

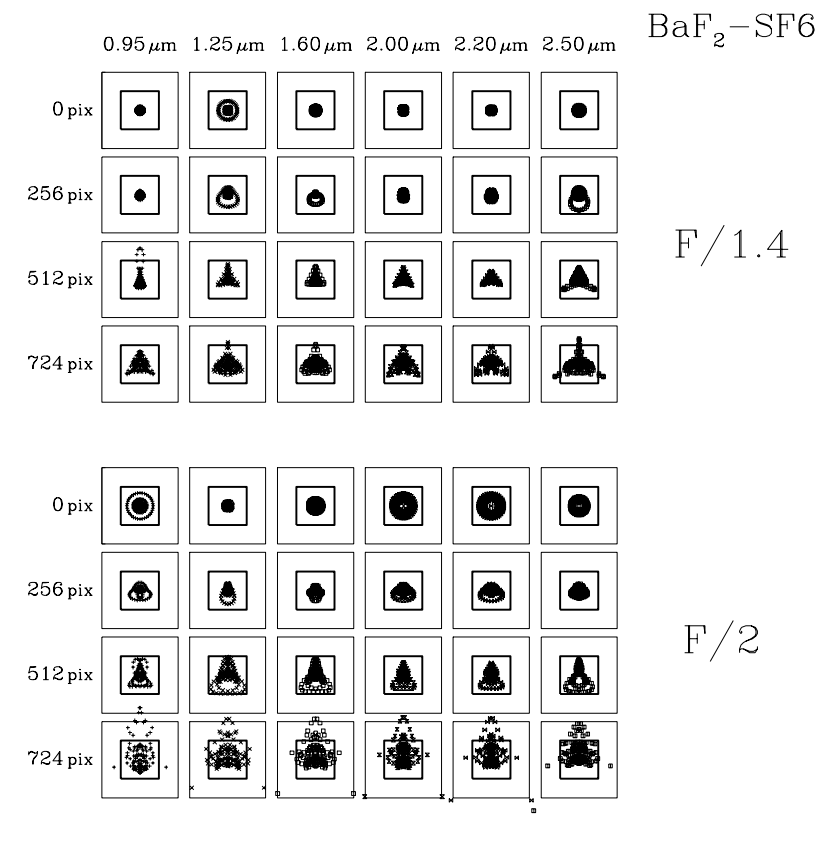

Fig. 4. Monochromatic spot diagrams (neglecting lateral chromatism) at several wavelengths and distances from the array center for the $\mathrm{BaF}_{2}-\mathrm{SF} 6$ cameras (Fig. 3), the array is a $1024^{2}$ with pixels of $18.5 \mu \mathrm{m}$. The size of one pixel is indicated by the thick squares, while the larger squares are 2 pixels $(37 \mu \mathrm{m})$

for lateral chromatism because this aberration does not influence the spectrograph performances.

The performances of the $\mathrm{BaF}_{2}-\mathrm{SF} 6$ cameras are remarkably good and virtually identical to those obtained using $\mathrm{BaF}_{2}-\mathrm{IRG} 2$. The $\mathrm{BaF}_{2}-\mathrm{SF} 56 \mathrm{~A}$ combination is also very good for $\lambda \lesssim 2.2 \mu \mathrm{m}$ but not at longer wavelengths where their chromatisms decouple, but this limitation is not important for applications to instruments not extending into the $\mathrm{K}$ atmospheric window. As already mentioned in Sect. 2, the image quality is not influenced by the exact behaviour of $\mathrm{d} n / \mathrm{d} T$ with wavelength, and any reasonable uncertainty on this parameter can be recovered by simply refocusing the array.

The design of the cameras was somewhat simplified by the use of one aspheric surface on $\mathrm{BaF}_{2}$, but this should not be a problem because this material can be quite easily diamond turned. Another potential limitation is the size of the $\mathrm{BaF}_{2}$ elements, especially in the $\mathrm{F} / 1.4$ camera which requires lenses with $D \gtrsim 180 \mathrm{~mm}$ useful diameters. However, these are not much larger than the $\oslash 160 \mathrm{~mm} \mathrm{BaF}_{2}$ aspheric collimator mounted in ISAAC, the ESO infrared instrument for the ESO-VLT $8 \mathrm{~m}$ telescope (Delabre 1993). We believe therefore that the designs presented here are within the present possibilities of lens manufacturers. 
Table 1. Refraction indices of $\mathrm{BaF}_{2}$ and Flint glasses

At $25{ }^{\circ} \mathrm{C}$

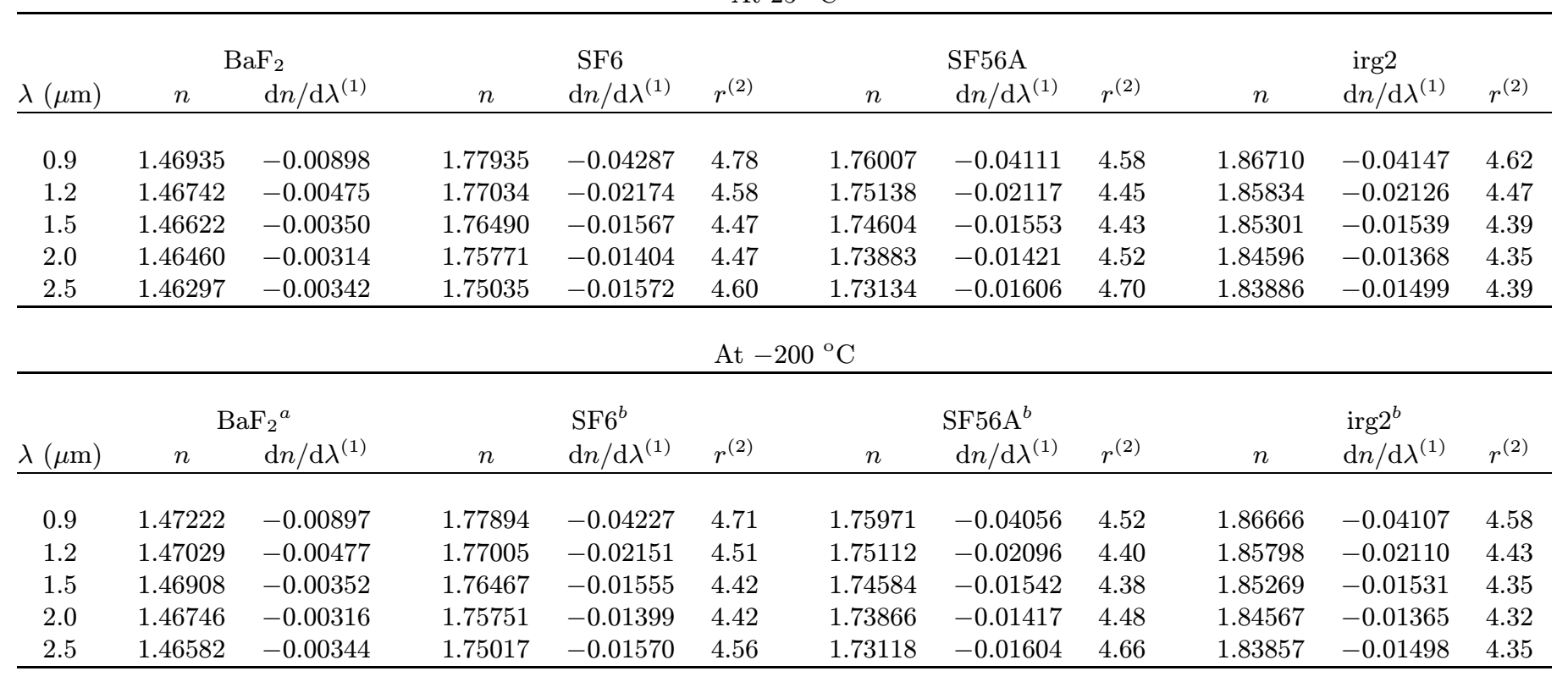

Notes to Table 1

(1) Dispersion, in $\mu \mathrm{m}^{-1}$.

(2) $r=$ glass dispersion $/ \mathrm{BaF}_{2}$ dispersion.

$a^{a}$ From Eq. (1), based on d $n / \mathrm{d} T$ from Feldman et al. (1979), cf. Sect. 2.

${ }^{b}$ Using the $D_{0}, D_{1}, D_{2}, E_{0}, E_{1}, \lambda_{\text {TK }}$ coefficients from the Schott catalog (cf. Eq. 2).

Table 2. Details of the cameras ${ }^{(1)}$

$$
\mathrm{F} / 2 \text { camera }
$$

F/1.4 camera

\begin{tabular}{|c|c|c|c|c|c|c|c|}
\hline Surface & Radius (mm) & Thickness (mm) & material & Surface & Radius (mm) & Thickness (mm) & material \\
\hline Source & $\infty$ & $\infty$ & \multirow{4}{*}{$\mathrm{BaF}_{2}$} & Source & $\infty$ & $\infty$ & \multirow{4}{*}{$\mathrm{BaF}_{2}$} \\
\hline Stop & $\infty$ & 200 & & Stop & $\infty$ & 200 & \\
\hline Lens 1 & 91.6531 & 40.00 & & Lens 1 & $-1843.86^{b}$ & 30.45 & \\
\hline Lens 1 & 1944.23 & 4.761 & & Lens 1 & -248.078 & 26.28 & \\
\hline Lens 2 & 605.277 & 7.823 & \multirow[t]{2}{*}{ SF6 } & Lens 2 & -489.278 & 10.00 & \multirow[t]{2}{*}{ SF6 } \\
\hline Lens 2 & 167.455 & 3.000 & & Lens 2 & 345.473 & 29.56 & \\
\hline Lens 3 & 116.127 & 26.62 & \multirow[t]{2}{*}{$\mathrm{BaF}_{2}$} & Lens 3 & -4226.46 & 49.88 & \multirow[t]{2}{*}{$\mathrm{BaF}_{2}$} \\
\hline Lens 3 & $152.138^{a}$ & 145.8 & & Lens 3 & -127.895 & 85.98 & \\
\hline Lens 4 & 57.9477 & 40.00 & \multirow[t]{5}{*}{ SF6 } & Lens 4 & 178.691 & 50.00 & \multirow[t]{2}{*}{$\mathrm{BaF}_{2}$} \\
\hline Lens 4 & 48.2670 & 15.00 & & Lens 4 & -348.386 & 71.84 & \\
\hline \multirow[t]{3}{*}{ array } & $\infty$ & 0 & & Lens 5 & 71.5761 & 46.59 & \multirow[t]{3}{*}{ SF6 } \\
\hline & & & & Lens 5 & 43.6675 & 19.41 & \\
\hline & & & & array & $\infty$ & 0 & \\
\hline
\end{tabular}

Notes to Table 2

(1) All values are at $-200{ }^{\circ} \mathrm{C}$.

${ }^{a}$ Aspheric, $A=3.21610^{-7}, B=5.42610^{-11}, C=-6.35210^{-15}, D=5.52610^{-18}$

$b$ Aspheric, $A=-1.33510^{-7}, B=-3.60610^{-12}, C=-2.59110^{-16}, D=2.88210^{-20}$. 


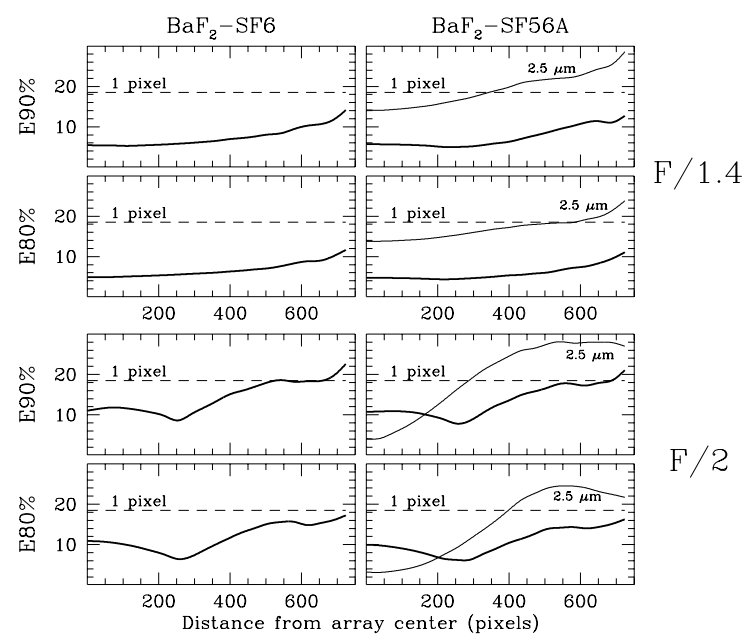

Fig. 5. Diameters of $80 \%$ and $90 \%$ encircled energies (in $\mu \mathrm{m}$ ) for the F/2 and F/1.4 cameras. The value is the maximum image size at the wavelengths of Fig. 4, for SF56A the curve at $2.5 \mu \mathrm{m}$ is shown separately. Note that $\mathrm{BaF}_{2}-\mathrm{SF} 6$ is excellent over the full range (and is in practice equivalent to $\mathrm{BaF}_{2}$-IRG2). The other pair is slightly better at the shortest $\lambda$ 's and is achromatic up to about $2.2 \mu \mathrm{m}$, but rapidly deteriorates beyond

\section{The IR transmission of SF glasses}

We performed measurements of SF5, SF6 and SF56A specimens using the Perkin-Elmer lambda9 system at the National Optical Institute of Florence. The resulting external transmission is plotted in the bottom panels of Fig. 6 where one can see that both glasses are basically transparent up to $1.35 \mu \mathrm{m}$, i.e. up to the edge of the $\mathrm{J}$ atmospheric window. Absorption features appear at longer wavelengths, a relatively narrow and symmetric line centered at $1.43 \mu \mathrm{m}$ followed by much broader absorption bands which become particularly strong beyond $2.1 \mu \mathrm{m}$.

The absorption coefficients are derived by normalizing the observed transmission to the level of the Fresnel's losses, and the results are shown in the upper panels of Fig. 6. These are in good agreement with the values derived from the internal transmission at laser wavelengths listed in the Schott catalog. The only significant discrepancy is for SF5 whose absorption coefficient at $1.53 \mu \mathrm{m}$ is similar to other glasses but a factor $\simeq 2$ larger than that reported by Schott.

Apart for the narrow absorption feature at $1.43 \mu \mathrm{m}$ (a wavelength where the atmosphere is basically opaque) the SF glasses considered here have negligible absorption, i.e. $a<0.01$, up to $1.65 \mu \mathrm{m}$ (the center of the $H$ atmospheric window) and $a \leq 0.02$ up to $1.8 \mu \mathrm{m}$, the long wavelength edge of the $\bar{H}$ band. In practice, this means that the fraction of light absorbed by the SF lenses in the cameras shown in Fig. 3 is $\leq 6 \%$ below $1.65 \mu \mathrm{m}$ and $\leq 12 \%$ between 1.65 and $1.8 \mu \mathrm{m}$. These small losses are relatively unimportant in astronomical instruments such

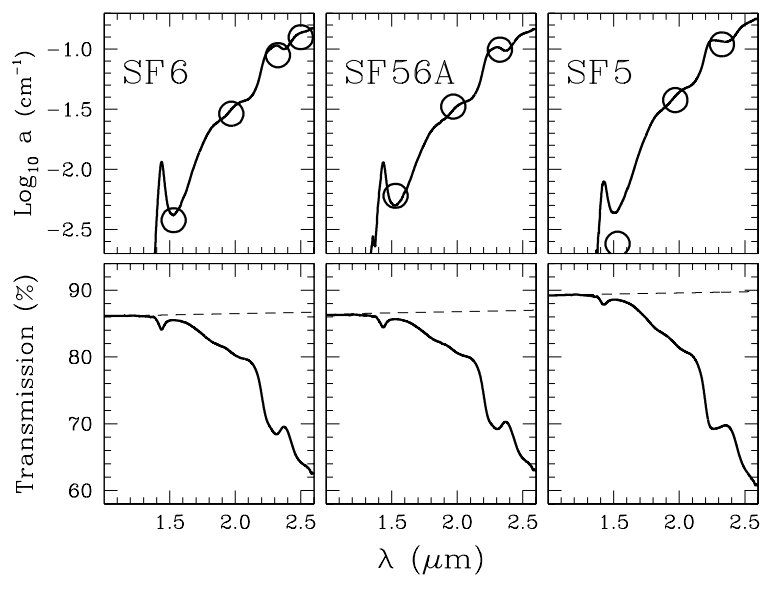

Fig. 6. Lower panels: measured external transmission of SF glass specimens, thickness $t=21.7 \mathrm{~mm}$. The dashed line represents the expected Fresnel's losses. Upper panels: derived absorption coefficients (internal transmission $=e^{- \text {at }}$ ), the open circles are the values derived from the Schott catalog

as fiber-fed spectrographs whose long wavelength transmission must be cut at about $1.7 \mu \mathrm{m}$ to avoid flooding the array with the thermal background from the warm parts of the instrument.

Beyond $2 \mu \mathrm{m}$ the SF glasses become quite opaque and cannot therefore be used in IR instruments extending to the $\mathrm{K}$ atmospheric window. However, the IR absorption bands are only due to water which is normally bound in the glass matrix, and can be eliminated by melting small cubicles of glass in vacuum environment, i.e. using the same fabrication process of FK54 and IR glasses (Knapp 1997). Clearly, this requires a special and expensive preparation which could be most conveniently supported by a large consortium of astronomers.

Acknowledgements. We are grateful to Konrad Knapp (Schott Glaswerke, Mainz) for many useful information on the SF and IRG glasses. We would like to thank A. van Dijsseldonk for helpful information, V. Castellini and D. Iafrancesco for their assistance with the Perkin-Elmer instrument, and A. Pecchioli (Gestione SILO, Florence) for preparing the SF specimens.

\section{References}

Delabre B., 1993, "The ISAAC optical design", ESO document No. VLT-TRE-ESO-14100-0488

Delabre B., 1994 (private communication)

Doyon R., Nadeau D., Vallee P., 1995, SPIE 2475, 184

Feldman A., Horowitz D., Waxler R.M., Dodge M.J., 1979, NBS Tecnical Report \# 993, p. 43

Knapp K., 1997 (private communication)

Oliva E., Gennari S., 1995, A\&AS 114, 179

van Dijsseldonk A., 1995 (private communication) 